\title{
Involvement of Transcription Factor 21 in the Pathogenesis of Fibrosis in Endometriosis
}

Umida Ganieva, ${ }^{*}$ Tomoko Nakamura, ${ }^{*}$ Satoko Osuka, ${ }^{* \dagger}$ Bayasula, ${ }^{\ddagger}$ Natsuki Nakanishi, ${ }^{*}$ Yukiyo Kasahara, ${ }^{*}$ Nobuyoshi Takasaki, Ayako Muraoka, ${ }^{*}$ Shotaro Hayashi, ${ }^{*}$ Takashi Nagai, ${ }^{*}$ Tomohiko Murase, ${ }^{*}$ Maki Goto, ${ }^{*}$ Akira Iwase, ${ }^{\S}$ and Fumitaka Kikkawa*

From the Bell Research Center for Reproductive Health and Cancer, ${ }^{\ddagger}$ Department of Obstetrics and Gynecology, * Nagoya University Graduate School of Medicine, Nagoya; the Department of Maternal and Perinatal Medicine, ${ }^{\dagger}$ Nagoya University Hospital, Nagoya; and the Department of Obstetrics and Gynecology, ${ }^{\S}$ Gunma University Graduate School of Medicine, Maebashi, Japan

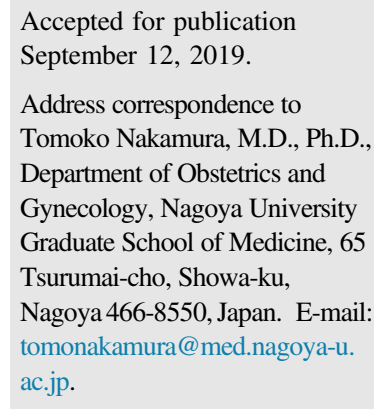

\begin{abstract}
Repeated tissue injury and repair and fibrosis play a pivotal role in endometriosis. Fibrotic tissue consists of extracellular matrix proteins, regulated by transcriptional factors promoting cell proliferation and survival. Periostin is one of the putative key extracellular matrix proteins. This study aimed to determine whether transcription factor 21 (TCF21) is involved in the development of endometriosis as an upstream regulatory gene of periostin. Formalin-fixed, paraffin-embedded tissue samples [normal endometrium of women without endometriosis; eutopic endometrium of women with endometriosis; ovarian endometriosis (OE); and deep infiltrating endometriosis (DIE)] and respective cells were analyzed. Basal, transiently stimulated, and knocked down periostin and TCF21 concentrations in stromal cells of women with or without endometriosis were examined. Periostin and TCF21 expressions were undetected in normal endometrium of women without endometriosis, weakly positive in eutopic endometrium of women with endometriosis, moderately positive in $0 \mathrm{E}$, and strongly positive in DIE. Type 2 helper T-cell cytokines (IL-4, IL-13, and transforming growth factor- $\beta 1$ ) increased the mRNA expression of periostin and TCF21. These cytokines, periostin, and TCF21 colocalized in the stroma of OE and DIE. siRNA against human TCF21 gene suppressed periostin expression. Transfection of TCF21 plasmid vector into stromal cells of women without endometriosis, which originally expressed neither periostin nor TCF21, resulted in TCF21 and periostin expression. TCF21 and periostin are involved in the regulation of fibrosis in endometriosis. TCF21 may be a promising therapeutic target and biomarker in endometriosis. (Am J Pathol 2020, 190: 145-157; https://doi.org/10.1016/j.ajpath.2019.09.008)
\end{abstract}

Repeated tissue injury and repair, resulting in fibrosis, may be one of the many pathologic mechanisms involved in endometriosis, which affects up to $15 \%$ of reproductive-age women. ${ }^{2}$ Endometriosis is often accompanied by a profound worsening of the quality of life because of concomitant chronic pain and infertility. ${ }^{3}$ Although it has been hypothesized that hormonal, immunologic, and environmental changes may induce normal endometrial cells to flow retrograde into the intraperitoneal space and become endometriotic, ${ }^{4}$ the detailed description of all of the abnormalities leading to endometriosis is yet to be elucidated.

Endometriosis is a benign disease going through tumor-like processes to form endometriotic lesions. Tumor-like processes include aggression, ${ }^{5}$ evasion [epithelial-mesenchymal transition (EMT)], ${ }^{6,7}$ adhesion (CD10 and integrins), ${ }^{8}$ invasion (matrix metalloproteinases), ${ }^{9}$ angiogenesis (vascular endothelial growth factor), ${ }^{10,11}$ surviving (hormones and aromatase), ${ }^{12}$ fibroproliferation, ${ }^{13}$ and inflammation (cytokines). ${ }^{14,15}$ Although these mechanisms have been studied for a long time, more research is still needed as the current medical therapies have many adverse effects and are not plausible because of the high recurrence rate after performing conservative surgery. ${ }^{16}$

Supported by Japan Society for the Promotion of Science grants-in-aid for scientific research $15 \mathrm{~K} 15603$ (A.I.), the College Women's Association of Japan Scholarship (Tokyo, Japan) (U.G.), and the Geta no hanao scholarship (Nagoya University, Nagoya, Japan) (U.G.).

Disclosures: None declared. 
A
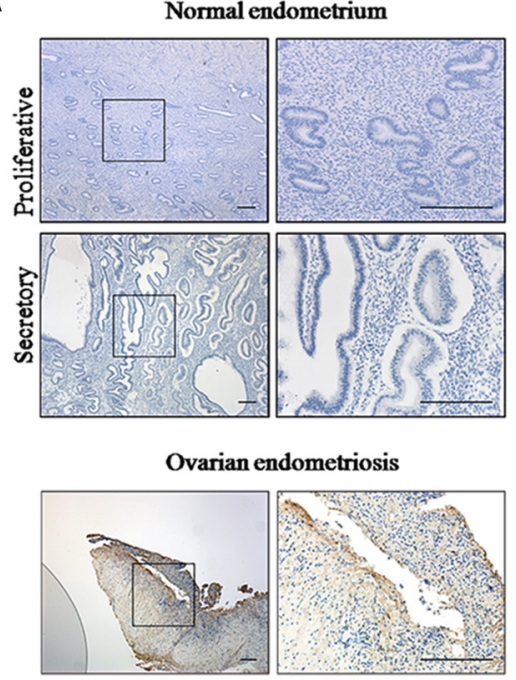

C

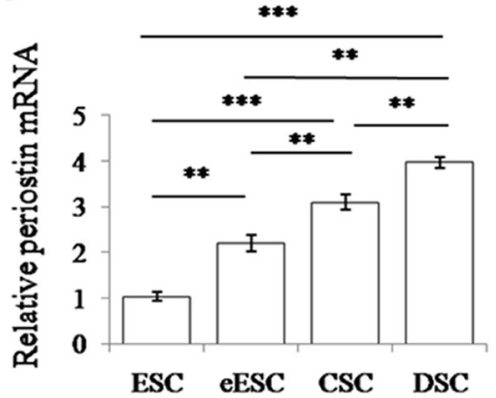

B

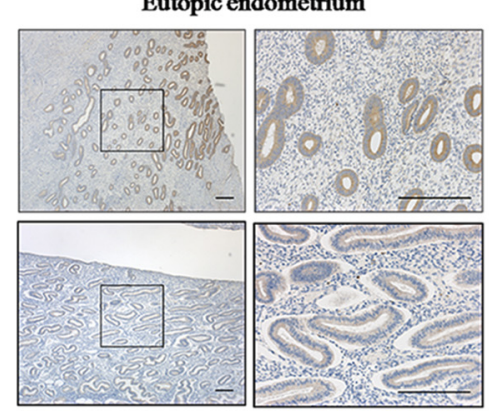

Deep infiltrating endometriosis

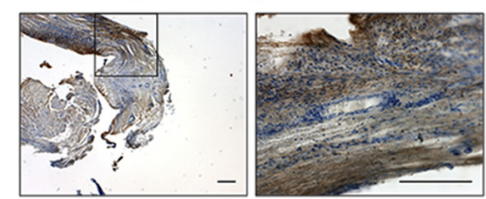

D

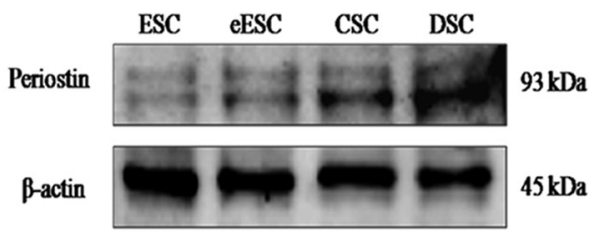

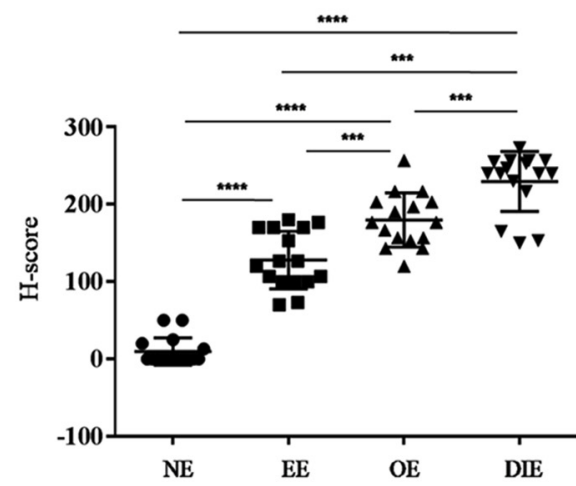

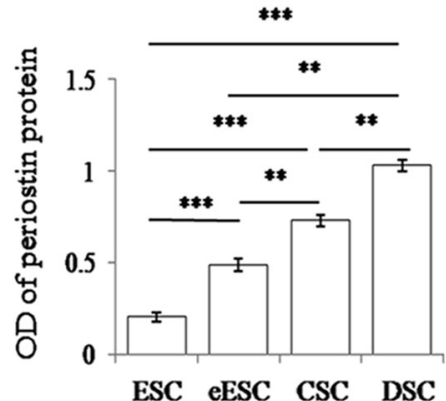

Figure 1 Levels of periostin in the samples from women with and without endometriosis. A: Immunohistochemical analysis of the levels of periostin protein expression. Boxed areas are shown at higher magnification to the right. B: Histo score (H-score) of the levels of periostin protein found in immunohistochemical analysis. C: Levels of periostin mRNA (48 hours). D: Western blot analysis of the levels of periostin protein (48 hours). The mean 0D ratio for periostin/ $\beta$-actin was calculated for each endometriotic cell type and compared with the $0 D$ ratio of the same proteins in normal endometrial cells. Data are expressed as means \pm SEM (B) or the means \pm SD (C and D). $\Sigma n=50(\mathbf{A}) ; \Sigma n=12$ (C and D). ${ }^{* *} P<0.01,{ }^{* * *} P<0.001$, and ${ }^{* * * * P<0.0001 . ~ S c a l e ~ b a r s: ~}$ $200 \mu \mathrm{m}$ (left columns); $50 \mu \mathrm{m}$ (right columns). Original magnification, $\times 5$ (left columns); $\times 20$ (right columns). CSC, chocolate cyst stromal cell; DIE, deep infiltrating endometriosis; DSC, deep infiltrating endometriosis stromal cell; EE, eutopic endometrium of women with endometriosis; eESC, eutopic ESC; $\mathrm{ESC}$, endometrial stromal cell; $\mathrm{NE}$, normal endometrium of women without endometriosis; $\mathrm{OE}$, ovarian endometriosis.

Extracellular matrix (ECM) proteins (fibronectin, tenascin-C, type I collagen, and laminin $\gamma 2$ ) play an important role in endometriosis. Periostin, a $90-\mathrm{kDa}$ osteoblast-specific factor-2 from the fasciclin family, ${ }^{17}$ functions as a scaffold for ECM protein assembly. ${ }^{18-20}$ On accumulation in the inflamed sites, periostin leads to fibrosis activating immune and nonimmune cells via its matricellular nature, by which it can bind to cell-surface receptors of integrins, cytokines, and other downstream signals, further expanding the inflammation. Physiologically, periostin is found at collagen-rich regions in connective tissue, such as periodontal ligament, ${ }^{21}$ cardiac valve, ${ }^{22}$ periosteum, ${ }^{23}$ and lung. ${ }^{24}$ Moreover, periostin is considered an important molecule involved in conditions associated with severe diseases, like fibrosis in asthma, ${ }^{25}$ scar formation in myocardial infarction, ${ }^{26}$ cancer cell migration, corneal dystrophy, skin, ${ }^{27}$ and endometriotic fibrosis. ${ }^{2-32}$ Therefore, periostin can be used for evaluation of fibrotic aspects of various tissues and endometriosis samples. ${ }^{25-32}$ Although the number of periostin-related articles in ophthalmology, dermatology, otolaryngology, allergology, and oncology is increasing every year, there are only few articles describing its expression and interaction with up-stream and down-stream targets in endometriosis. ${ }^{28-32}$

The gene coding for transcription factor $21, T C F 21$, is located on chromosome $6 \mathrm{q} 23$. It is a member of the basic helix-loop-helix transcription factor family and is essential for the epithelial cell differentiation. TCF21 is expressed in numerous tissues, including lung, gut, gonad, urinary tract, spleen, and kidney. ${ }^{33}$ In physiological conditions, TCF21 remains inactivated; and only if the tissue needs to be recovered after an injury or a stress, it becomes aberrantly activated. ${ }^{34-36}$ The transcription factors of the basic helixloop-helix family regulate the expression of hundreds of other genes, including those promoting cell proliferation 

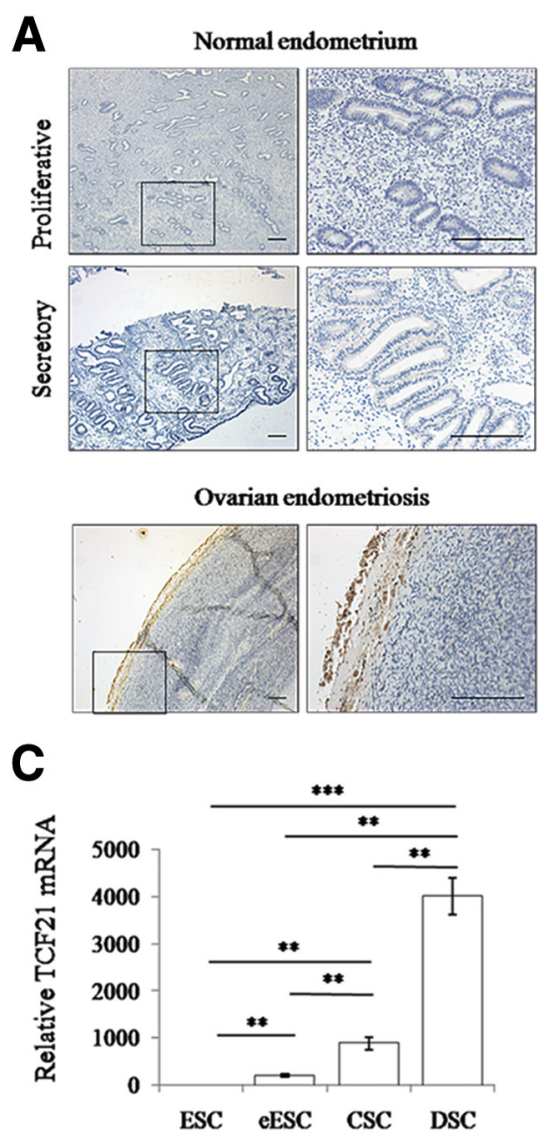
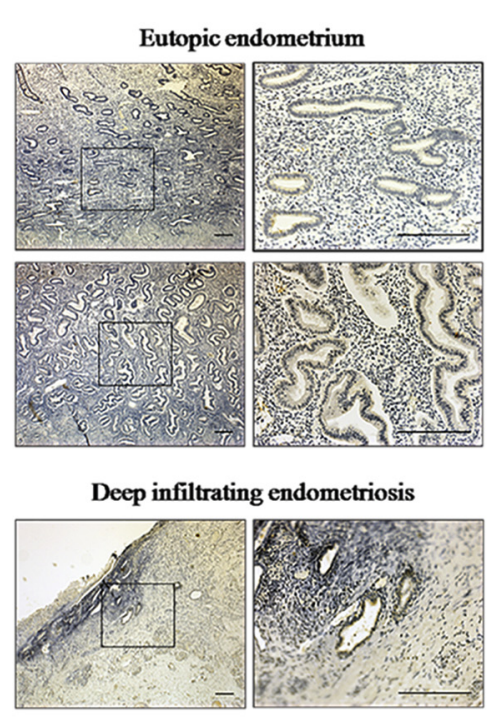

D

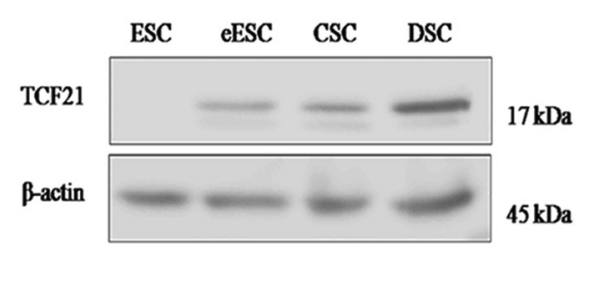

B
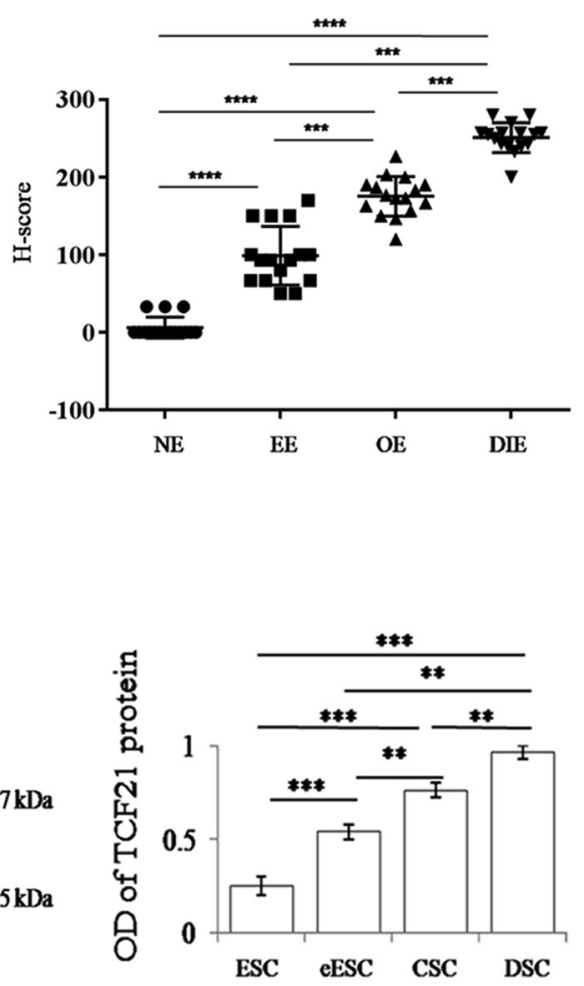

Figure 2 Levels of TCF21 in the samples from women with and without endometriosis. A: Immunohistochemical analysis of the levels of TCF21 protein expression. Boxed areas are shown at higher magnification to the right. B: Histo score (H-score) of the levels of TCF21 protein found in immunohistochemical analysis. C: Levels of TCF21 mRNA (48 hours). D: Western blot analysis of the levels of TCF21 protein (48 hours). The mean 0D ratio for TCF21/ $\beta$-actin was calculated for each endometriotic cell type and compared with the $0 \mathrm{D}$ ratio of the same proteins in normal endometrial cells. Data are expressed as means \pm SEM (B) or the means \pm SD (C and D). $\Sigma n=50(\mathbf{A}) ; \Sigma n=12$ (C and D). ${ }^{* *} P<0.01,{ }^{* * *} P<0.001$, and ${ }^{* * * *} P<0.0001$. Scale bars: $200 \mu \mathrm{m}$ (left columns); $50 \mu \mathrm{m}$ (right columns). Original magnification, $\times 5$ (left columns); $\times 20$ (right columns). CSC, chocolate cyst stromal cell; DIE, deep infiltrating endometriosis; DSC, deep infiltrating endometriosis stromal cell; EE, eutopic endometrium of women with endometriosis; eESC, eutopic ESC; ESC, endometrial stromal cell; $\mathrm{NE}$, normal endometrium of women without endometriosis; $0 \mathrm{E}$, ovarian endometriosis.

and survival, and EMT via $\mathrm{Wnt} / \beta$-catenin signaling. Although many studies have focused on the role of $\mathrm{Wnt} / \beta$ catenin pathway ${ }^{37,38}$ and on its silencing effect in endometriosis, ${ }^{39}$ up to date, no study has demonstrated the direct interaction between $T C F 21$ and periostin in endometriosis. Studies on the role of TCF21 and periostin in myocardial infarction showed that TCF21 is an upstream regulatory gene of periostin and TCF 21 becomes activated from the resident fibroblasts, leading to the overproduction of its downstream target, periostin. ${ }^{34,35}$

Our aim was to investigate whether TCF21 is involved in the regulation of periostin in endometriosis. We demonstrate how TCF21 regulation affects periostin expression and, therefore, the pathogenesis of endometriosis. This study provides an insight into the expression of TCF21, periostin, and cytokines, and their interaction in the samples from women with or without endometriosis, and further substantiates the value of TCF21 as a therapeutic target and putative biomarker in endometriosis.

\section{Materials and Methods}

\section{Reagents}

The reagents used were as follows: antiperiostin antibody (ab92460), anti-TCF21 antibody-chromatin immunoprecipitation grade (ab32981), anti-IL-4 antibody (EPR1118Y; ab62351), anti-transforming growth factor (TGF)- $\beta 1$ antibody (ab92486; Abcam, Cambridge, UK), anti-IL-13 antibody (F-6; sc-390676; Santa Cruz Biotechnology, Inc., Dallas, TX), anti- $\beta$-actin antibody (Wako Pure Chemical Industries, Ltd., Osaka, Japan), clone OTI4C5 anti-DDK (Flag) monoclonal antibody (TA50011100; OriGene Technologies, Inc., Rockville, MD), Opal Multiplex IHC Detection Kit (PerkinElmer, Waltham, MA); recombinant cytokines (PeproTech, Rocky Hill, NJ) human IL-4 (catalog number AF-200-04), human IL-13 (catalog number AF-200-13), and human TGF- $\beta 1$ (catalog number AF-100-21C); Lipofectamine 3000 and Lipofectamine RNAiMAX (Invitrogen, Waltham, MA); TCF21 
A

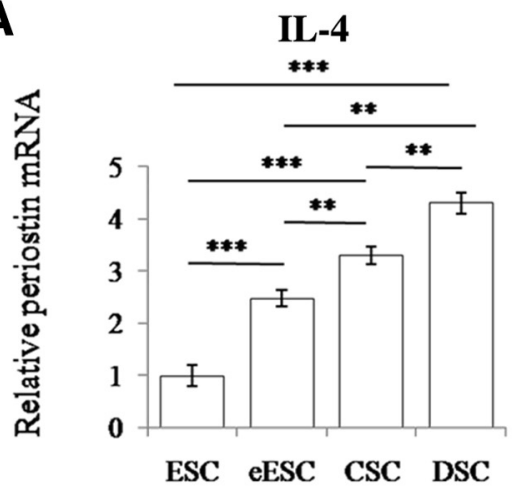

B

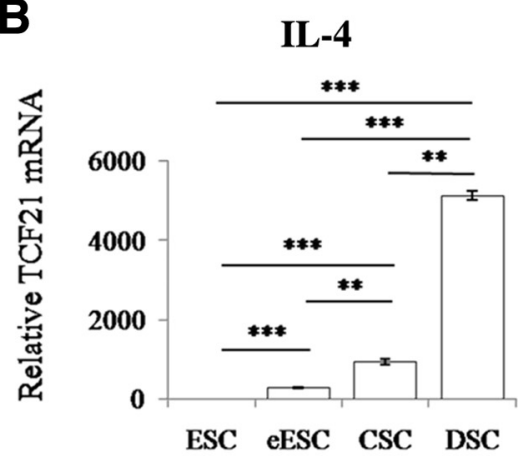

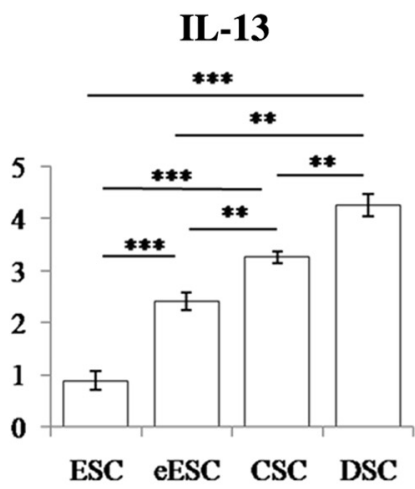

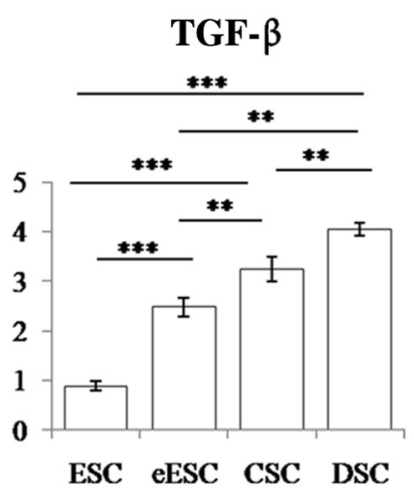

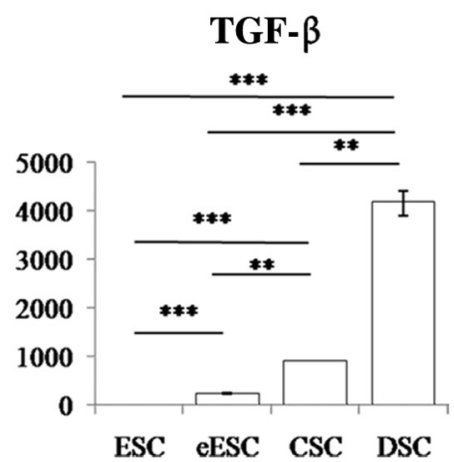

Figure 3 Effect of cytokines on periostin and TCF21 mRNAs. A: Levels of periostin mRNA in the samples from women with and without endometriosis after stimulation with cytokines (48 hours). B: Levels of TCF21 mRNA in the samples from women with and without endometriosis after stimulation with cytokines (48 hours). Data are expressed as means \pm SEM (A and $\mathbf{B}) . \Sigma n=12$ (A and $\mathbf{B}) .{ }^{* *} P<0.01,{ }^{* *} P<0.001$. CSC, chocolate cyst stromal cell; DSC, deep infiltrating endometriosis stromal cell; eESC, eutopic ESC; ESC, endometrial stromal cell; TGF- $\beta$, transforming growth factor- $\beta$.

silencer pool (Ambion, Life Technologies, Carlsbad, CA; s13904, s13906, s224713, and s13905) and non-specific control siRNA (Invitrogen); TCF21 (https://www.ncbi. nlm.nih.gov/nuccore; accession number NM_198392) Human cDNA ORF Clone (OriGene Technologies, Inc.) and empty plasmid (OriGene Technologies, Inc.; https:// www.ncbi.nlm.nih.gov/nuccore; accession number $\mathrm{NM}_{-}$ 004098). Green fluorescent protein- and Flag-tagged TCF21 vector plasmids were generated for the transfection into normal endometrial cells. Protein-level and cytologic localization of TCF21 and periostin were evaluated by Western blot (WB) and immunocytochemical analysis.

\section{Tissue Samples}

A total of 50 formalin-fixed, paraffin-embedded (FFPE) tissue samples of women with or without endometriosis (5 $\mu \mathrm{m}$ thick sections) were used: NE, normal endometrium without endometriosis, $n=14$; EE, eutopic endometrium with endometriosis, $n=11$; OE, ovarian endometriosis, $n=16$; and DIE, deep infiltrating endometriosis, $n=9$. The ethical committee of Nagoya University Graduate School of Medicine (Nagoya, Japan) approved the experiments. Written informed consent was obtained from each patient before sampling.

\section{Cell Culture}

Stromal cells were obtained from control endometrium $(n=6)$ of patients undergoing surgery for fibroid or other benign gynecologic diseases and from eutopic $(n=7)$ and ectopic endometrium of women with OE $(n=7)$ and DIE $(n=3)$. The cells were confirmed by a pathologist to be normal endometrial [endometrial stromal cell (ESC)], eutopic (eutopic ESC), and ectopic stromal cells [chocolate cyst stromal cells (CSCs) and deep infiltrating endometriosis stromal cells (DSCs)]. The patients did not receive any hormonal treatment at least 6 months before surgery. Briefly, biopsy specimens were rinsed by phosphate-buffered saline, minced into $1-\mathrm{mm}^{3}$ pieces, and digested with $5 \%$ dispase and collagenase $(2 \mathrm{mg} / \mathrm{mL}$; Gibco Invitrogen, Cergy Pontoise, France) for 1 hour at $37^{\circ} \mathrm{C}$, with serial filtration for separation. Hypotonic lysis buffer was used to remove red blood cells. Undigested tissue was removed using $100-\mu \mathrm{m}$ sieves. The stromal cells were further enriched by separation of epithelial cells with a $40-\mu \mathrm{m}$ sieve. Isolated stromal cells were cultured in Dulbecco's modified Eagle's medium supplemented with $10 \%(\mathrm{v} / \mathrm{v})$ fetal bovine serum (Gibco-BRL, Gaithersburg, $\mathrm{MD}), 100 \mathrm{IU} / \mathrm{mL}$ of penicillin, $100 \mu \mathrm{g} / \mathrm{mL}$ of streptomycin, and $0.025 \mathrm{mg} / \mathrm{mL}$ of amphotericin $\mathrm{B}$, at $37^{\circ} \mathrm{C}$ in $5 \%(\mathrm{v} / \mathrm{v}) \mathrm{CO}_{2}$ in air. To confirm the correct stromal phenotype, the isolated cells were checked for their positivity for vimentin (1:50 


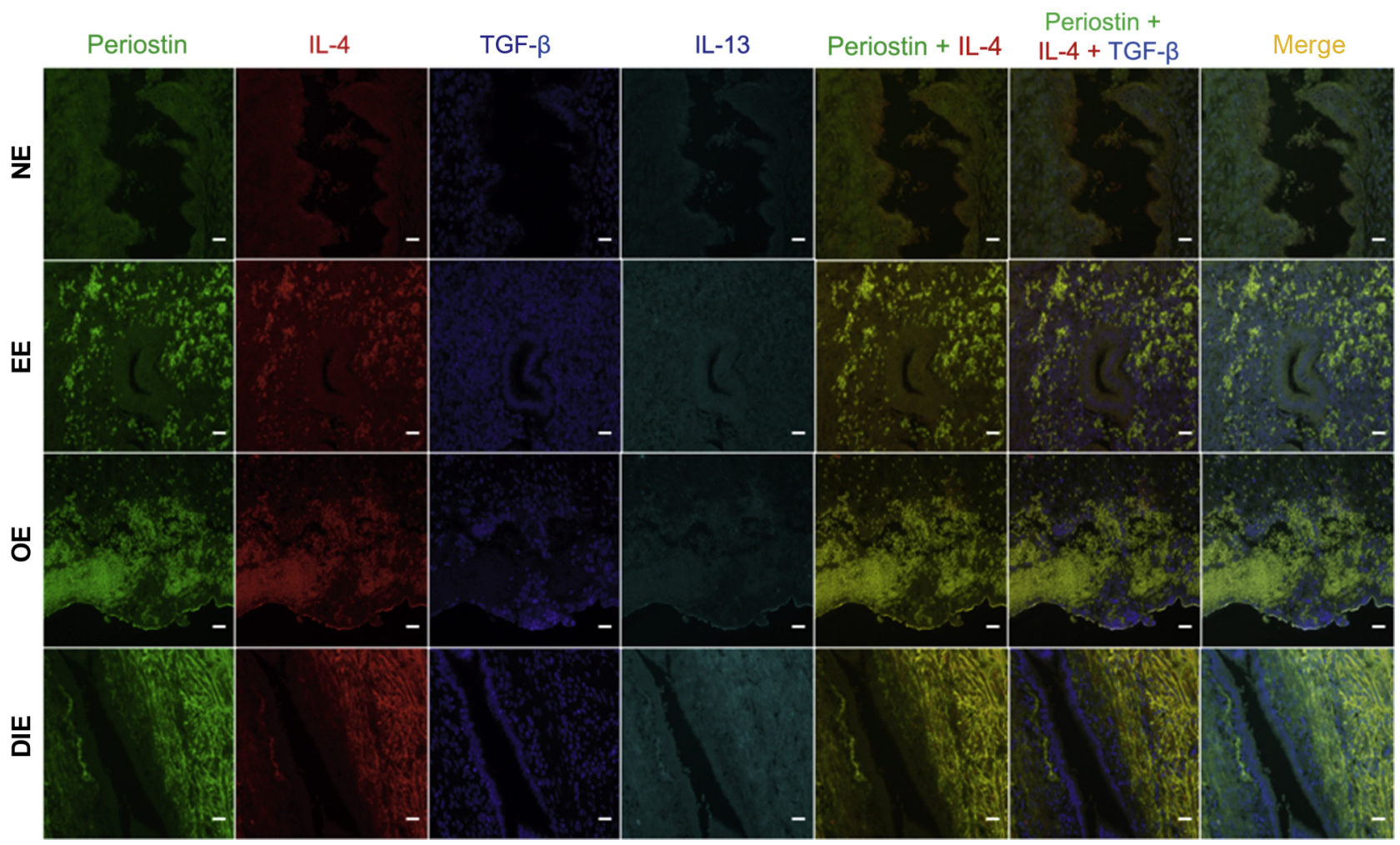

Figure 4 Expression and colocalization of periostin and cytokines shown by immunofluorescence. $\Sigma n=50$. Scale bars $=200 \mu \mathrm{m}$. Original magnification, $\times 40$. DIE, deep infiltrating endometriosis; $E$, eutopic endometrium of women with endometriosis; $N E$, normal endometrium of women without endometriosis; $O E$, ovarian endometriosis; TGF- $\beta$, transforming growth factor- $\beta$.

antivimentin; Cell Signaling Technology, Danvers, MA) and for their negative epithelial phenotype using cytokeratin (1:25 anticytokeratin; Cell Signaling Technology) by cell immunofluorescence. The monolayer culture cells, after the third passage, were $>99 \%$ pure (the proportion of stromal cells was evaluated in five random images using $\times 20$ magnification). Each experiment was performed in triplicate and repeated at least three times with cells isolated from separate patients.

\section{Immunohistochemistry}

Formalin-fixed human specimens (5 $\mu \mathrm{m}$ thick) were deparaffinized, blocked in methanol/ $0.3 \% \mathrm{H}_{2} \mathrm{O}_{2}$, and incubated with polyclonal primary antibodies against periostin and TCF21 (1:500; Abcam) overnight at $4^{\circ} \mathrm{C}$, followed by incubation with horseradish peroxidase-conjugated secondary antibody. Then, all specimens were incubated with 3,3'-diaminobenzidine tetrahydrochloride (Dako, Glostrup, Denmark), counterstained with hematoxylin, dehydrated, and mounted. Positive and negative control slides were incorporated in each staining. Negative control slides were incubated with phosphate-buffered saline replacing the primary antibody. For semiquantification, each specimen was analyzed by counting periostin- and TCF21-positive cells in the epithelium and in the stroma, by using Zeiss ZEN 2 (blue edition) microscope and Zeiss software version 1.0en.3.1 (Carl Zeiss Microscopy GmbH, Jena, Germany). The histo score was calculated by assigning a four-point scale to the intensity of the staining in 10 random fields ( 0 indicates negative; 1 , weak; 2 , moderate; and 3, strong); the percentage of cells at each intensity level was calculated by the following formula: $\left[1 \times\left(\%\right.\right.$ cells $\left.1^{+}\right)+2 \times\left(\%\right.$ cells $\left.2^{+}\right)+3 \times\left(\%\right.$ cells $\left.\left.3^{+}\right)\right]{ }^{28}$

\section{Measurement of the Protein Expression of Periostin and TCF21}

The cells were lysed in a radioimmunoprecipitation buffer $(10 \mathrm{mmol} / \mathrm{L}$ Tris-HCl, pH 7 to $4,150 \mathrm{mmol} / \mathrm{L} \mathrm{NaCl}, 1 \%$ Nonidet P-40, $5 \mathrm{mmol} / \mathrm{L}$ EDTA, $1 \%$ sodium deoxycholate, $0.1 \%$ SDS, $1.2 \%$ aprotinin, $5 \mu \mathrm{mol} / \mathrm{L}$ leupeptin, $4 \mu \mathrm{mol} / \mathrm{L}$ antipain, $1 \mathrm{mmol} / \mathrm{L}$ phenylmethylsulfonylfluoride, and 0.1 $\mathrm{mmol} / \mathrm{L} \quad \mathrm{Na}_{3} \mathrm{VO}_{4}$ ). The cell lysates were clarified by centrifugation at $13,000 \times g$ at $4^{\circ} \mathrm{C}$ for 15 minutes, for protein extraction. Equal amounts of proteins were mixed with $2 \times$ sample buffer (4\% SDS), $10 \% \beta$-mercaptoethanol, and $20 \%$ glycerol in $0.125 \mathrm{~mol} / \mathrm{L}$ Tris, $\mathrm{pH} 6.8$, containing bromophenol blue and boiled for 5 minutes. Equal amounts of proteins $(30 \mu \mathrm{g})$ were loaded and separated by $10 \%$ SDSPAGE for the analysis of periostin and TCF21 protein level. Blocking was done with 5\% skimmed milk for 1 hour at room temperature. Then, the membranes were incubated overnight at $4^{\circ} \mathrm{C}$ with antiperiostin (1:1000), anti-TCF21 antibody-chromatin immunoprecipitation grade ab32981 $(0.5 \mu \mathrm{g} / \mathrm{mL})$, and anti- $\beta$-actin antibody (1:4000) in $5 \%$ 


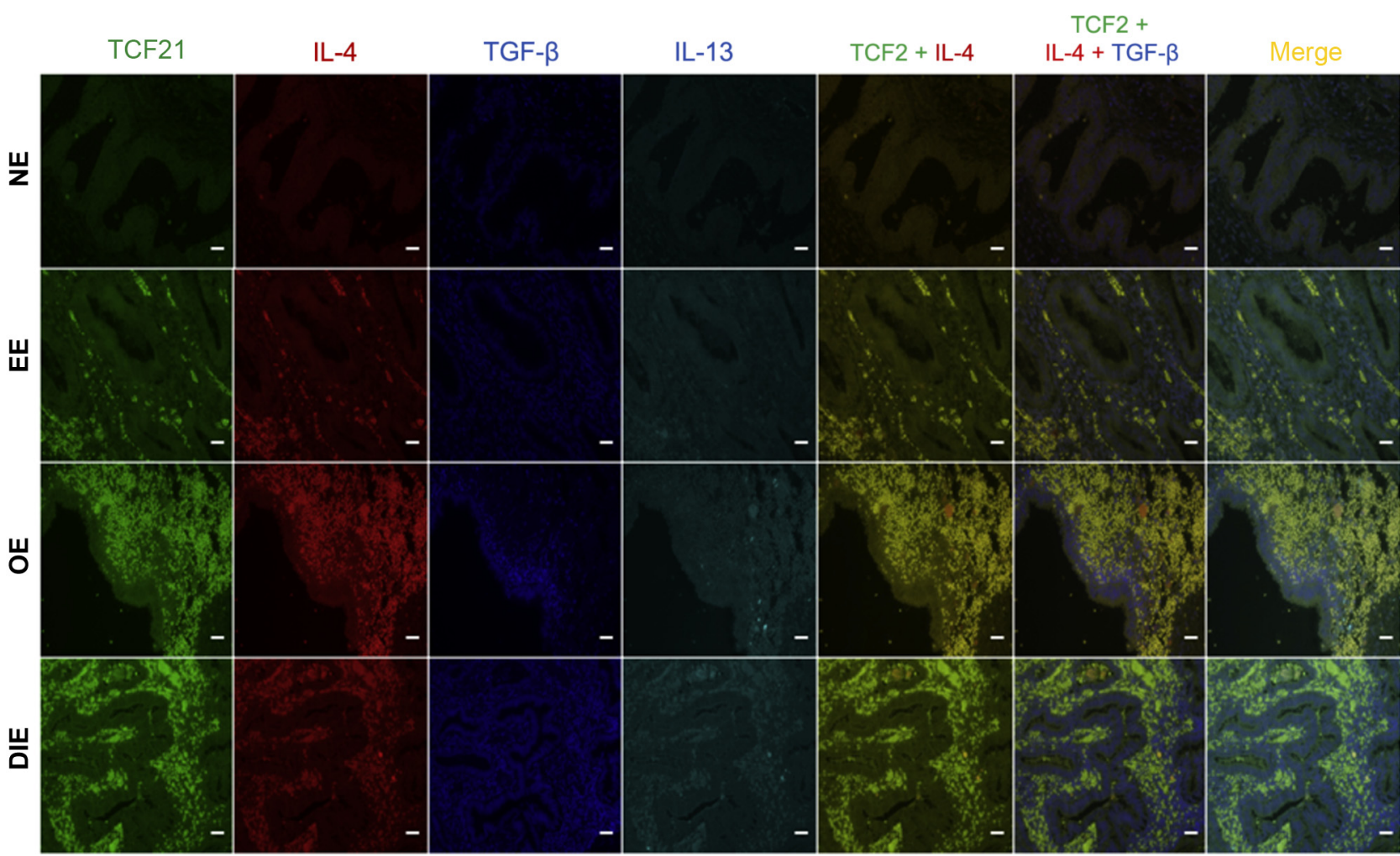

Figure 5 Expression and colocalization of TCF21 and cytokines shown by immunofluorescence. $\Sigma n=50$. Scale bars $=200 \mu \mathrm{m}$. Original magnification, $\times 40$. DIE, deep infiltrating endometriosis; $E$, eutopic endometrium of women with endometriosis; $N E$, normal endometrium of women without endometriosis; $\mathrm{OE}$, ovarian endometriosis; TGF- $\beta$, transforming growth factor- $\beta$.

nonfat dry milk/0.05\% phosphate-buffered saline-Tween. The binding of the specific antibodies was detected using a 1:1000 dilution of horseradish peroxidase-conjugated goat anti-rabbit (antiperiostin and anti-TCF21 antibodies) and goat anti-mouse (anti- $\beta$-actin antibody) $\operatorname{IgG}$ secondary antibody (Dako). $\beta$-Actin was used as a loading control. The membranes were visualized by ImageQuant LAS 4000 (GE Healthcare, Buckinghamshire, UK) after reaction with Amersham ECL WB detecting reagent (GE Healthcare). ODs were measured using ImageQuantTL (GE Healthcare). Ratios between periostin OD and $\beta$-actin OD or TCF21 OD and $\beta$-actin OD were calculated in each case.

\section{RNA Extraction and $q P C R$}

Total RNA was extracted from ESC, eutopic ESC, CSC, and DSC samples, using RNeasy Mini Kit (Qiagen, Tokyo, Japan). The RNA was also isolated from the same cells after stimulation with $20 \mathrm{ng} / \mathrm{mL}$ of cytokines, to verify whether they affect periostin and TCF 21 mRNA expression (type 2 helper $\mathrm{T}$ cell/profibrotic: IL-4, IL-13, and TGF- $\beta 1$ ). The RNA (10 ng) was then retrotranscribed using ReverTrace qPCR RT Master Mix (TOYOBO, Osaka, Japan), and the cDNA was used for performing real-time quantitative PCR (qPCR), which was performed in triplicate using LightCycler 480 (Roche Diagnostics, Penzberg, Germany) and KOD SYBR qPCR Mix (TOYOBO). The primers used for periostin were as follows: 5'-AATCCAAGTTGTCCCAAGCC- $3^{\prime}$ (forward) and $5^{\prime}$-GCACTCTGGGCATCG TGGGA-3' (reverse); for TCF21: 5'-TCCTGGCTAACGACAAATACGA-3' (forward) and 5'-TTTCCCGGC CACCATAAAGG-3' (reverse); and for Gapdh: $5^{\prime}$-CAGCC TCAAGATCATCAGCA-3' (forward) and 5'-GTCTTCT GGGTGGCAGTGAT-3' (reverse) (Thermo Fisher Scientific, Waltham, MA). Gapdh was used as internal control. Periostin and TCF21 expression levels were calculated relative to Gapdh by applying the $\Delta \Delta \mathrm{C}_{\mathrm{T}}$ method, and the data are presented as the percentage of periostin/Gapdh and TCF21/Gapdh of eutopic ESC, CSC, and DSC relative to that of ESC.

\section{Multiplexed Immunohistochemistry/ Immunofluorescence}

Multiplexed staining was performed using the Opal Multiplex IHC Detection Kit (PerkinElmer) following the manufacturer's protocol. Briefly, the slides were first deparaffinized in xylene and then rehydrated in ethanol, following antigen retrieval in citrate buffer (pH 6.0) using a microwave. Primary antibody for periostin (1:500) was incubated for 1 hour in a humidified chamber at room temperature, followed by rabbit Opal Polymer horseradish peroxidase mouse + rabbit secondary antibody working solution. Visualization of periostin was performed using 


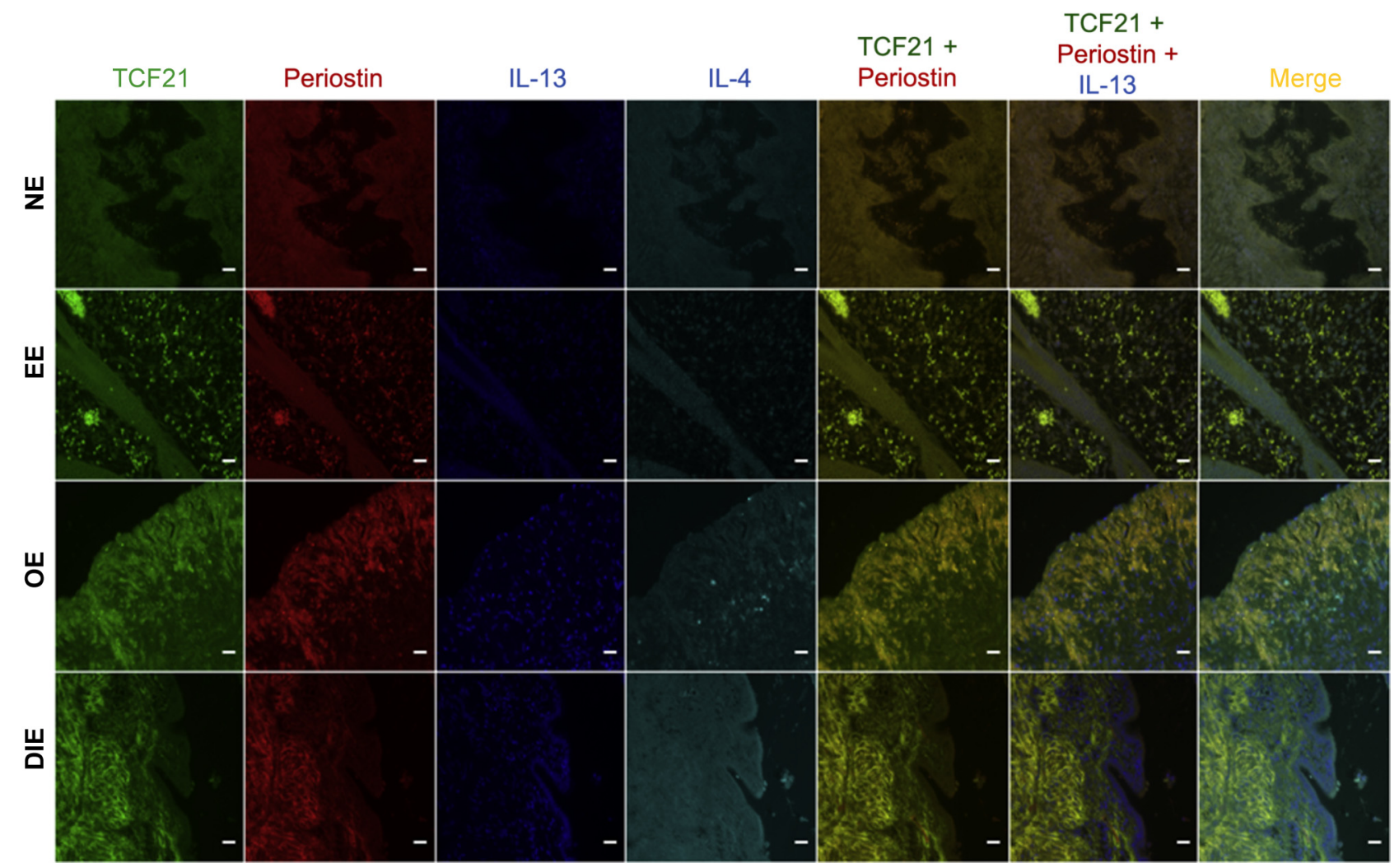

Figure 6 Expression and colocalization of TCF21, periostin, and cytokines shown by immunofluorescence. $\Sigma n=50$. Scale bars $=200 \mu \mathrm{m}$. Original magnification, $\times 40$. DIE, deep infiltrating endometriosis; EE, eutopic endometrium of women with endometriosis; NE, normal endometrium of women without endometriosis; $\mathrm{OE}$, ovarian endometriosis.

Opal 520 (GFP-B) TSA Plus (1:100; PerkinElmer), followed by treatment with citrate buffer $(\mathrm{pH} 6.0)$ and heating using a microwave. Each slide was then incubated in a serial manner, with primary rabbit antibodies for IL-4 (1:500) and TGF- $\beta 1$ (1:100) and mouse antibody for IL-13 (1:100) for 1 hour in a humidified chamber, at room temperature, followed by detection using the Opal Polymer horseradish peroxidase mouse + rabbit secondary antibody working solution (PerkinElmer). The expression of the cytokines IL4 , TGF- $\beta 1$, and IL- 13 was visualized using Opal 570 (Texas Red; PerkinElmer), Opal 690 (Cy5; PerkinElmer), and spectral DAPI (DAPI-B) TSA Plus (1:100; PerkinElmer) fluorophores, respectively. The same procedure was performed with TCF21 (Opal 520 fluorophore; $5.0 \mu \mathrm{g} / \mathrm{mL}$ ), IL4 (Opal 570 fluorophore), IL-13 (Opal 690 fluorophore), and TGF- $\beta 1$ (spectral DAPI fluorophore) primary antibodies in a serial manner, on the same slides. A third visualization was performed after applying TCF21 (Opal 520 fluorophore), periostin (Opal 570 fluorophore), IL-4 (Opal 690 fluorophore), and IL-13 (spectral DAPI fluorophore) on the same slides, in a serial mode. In all experiments, nuclei were subsequently visualized with DAPI working solution, and the sections were coverslipped using fluorescence mounting medium (Dako Agilent, Santa Clara, CA). The advantage of using the Opal method is that it allows applying four primary antibodies sequentially on a single slide.
Transient Knockdown and Overexpression of TCF21 Expression and Transfection

TCF21 was transiently knocked down by transfecting 10 nmol/L of siRNA against human TCF 21 gene (siTCF21 ID\#s13904) and non-specific control siRNA (Invitrogen) into CSCs and DSCs for 48 hours using Lipofectamine RNAiMAX, according to the manufacturer's instructions. For transient overexpression of TCF21, ESCs were transfected with $5 \mu \mathrm{g}$ Flag-tagged TCF21 plasmid and $5 \mu \mathrm{g}$ empty plasmid serving as control (OriGene Technologies, Inc.) for 48 hours using Lipofectamine 3000, according to the manufacturer's instructions.

\section{Immunocytochemistry}

ESCs were cultured on coverslips, transfected with TCF 21-Flag-tagged vector plasmid or empty plasmid serving as control for 48 hours, fixed in $4 \%$ paraformaldehyde for 15 minutes at room temperature, and permeabilized with $0.5 \%$ Triton $\mathrm{X}-100$ for 10 minutes. After blocking with 3\% bovine serum albumin for 30 minutes at room temperature, the cells were incubated with the primary antibodies (antiperiostin, anti-TCF21, and anti-DDK) at $4{ }^{\circ} \mathrm{C}$ overnight. Then, the cells were incubated with goat anti-rabbit 568 and 488 (Alexa 

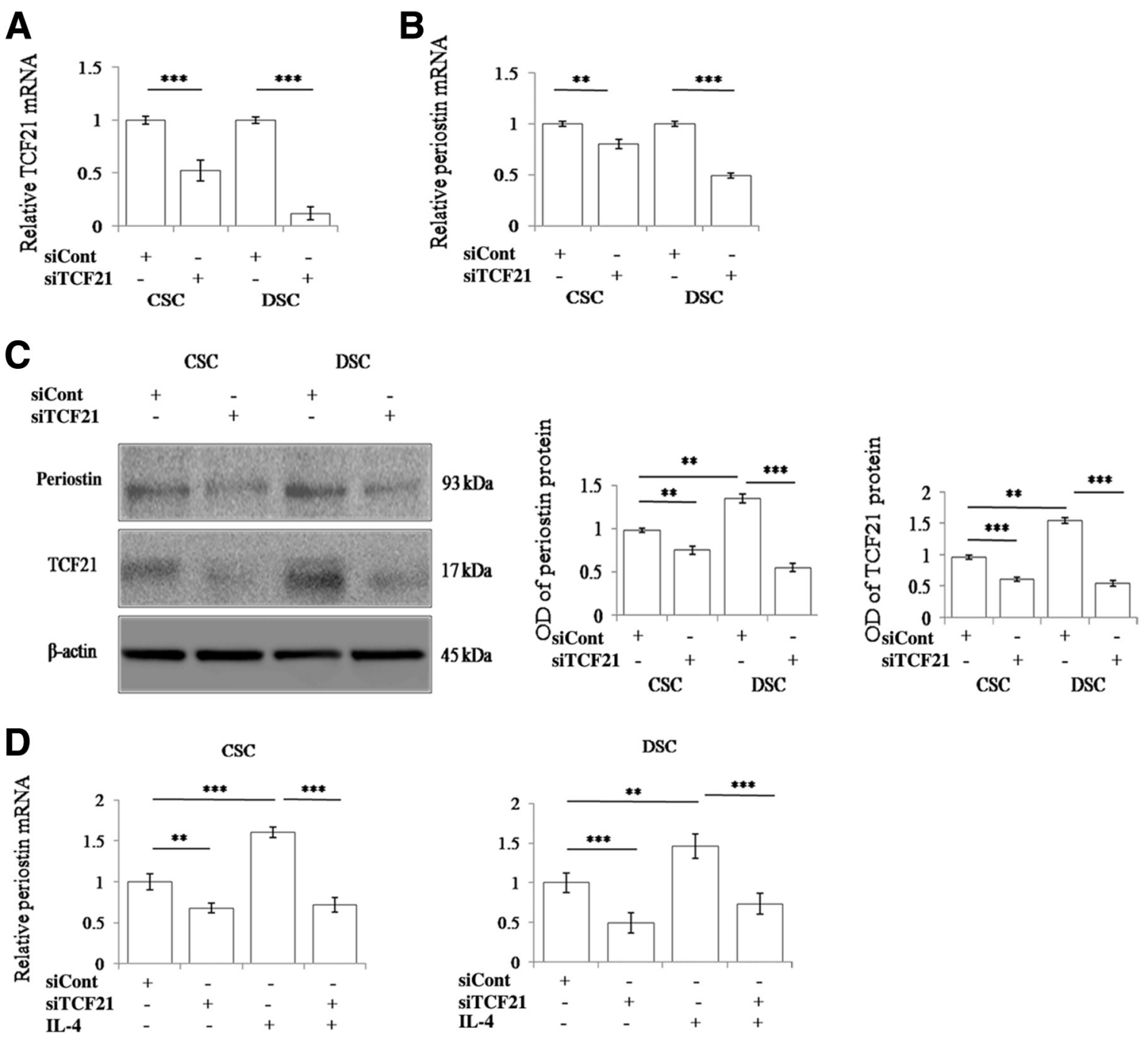

Figure 7 Effect of TCF21 siRNA on TCF21 and periostin. A: mRNA levels of TCF21 in chocolate cyst stromal cells (CSCs) and deep infiltrating endometriosis stromal cells (DSCs) after transfection with TCF21 siRNA (siTCF21; 48 hours). B: mRNA levels of periostin in CSCs and DSCs after transfection with TCF21 siRNA (48 hours). C: Western blot analysis of the levels of periostin protein after TCF21 siRNA transfection (48 hours). D: mRNA levels of periostin in CSCs and DSCs after TCF21 siRNA transfection and cytokine stimulation (48 hours). Data are expressed as means \pm SD. $n=3$ (A, B, and D, CSCS and DSCs); $\Sigma n=6$ (C). $* * P<0.01, * * * P<0.001$. siCont, non-specific control siRNA.

Fluor; Abcam; in case of antiperiostin and anti-TCF21 antibodies) and goat anti-mouse 488 (Alexa Fluor; Abcam; in case of anti-DDK antibody) secondary antibodies for 1 hour at room temperature. Hoechst 33342 (APExBIO Technology LLC, Houston, TX) was used for nuclei staining. Visualization was done by a confocal Laser Scanning Microscope (TiE-A1R; Nikon, Tokyo, Japan).

\section{Statistical Analysis}

Data were expressed as means \pm SEM or the means \pm SD. The level of significance between groups was determined by $t$-test or $U$-test. The $U$-test was applied instead of the $t$-test when the variables were not normally distributed. Statistical analyses were performed using SPSS software version 24.0 (SPSS Inc., Chicago, IL). The histo score was analyzed by
GraphPad Prism software version 7.01 (GraphPad Software, San Diego, CA). $P<0.05$ was considered statistically significant. All the experiments were performed at least in triplicate.

\section{Results}

\section{Periostin Expression}

To understand the role of periostin in endometriosis, its protein expression levels were assessed in the samples of women with or without endometriosis by immunohistochemistry. In NE, periostin was neither expressed in proliferative nor secretory phases of the menstrual cycle (Figure 1A). Weak expression with glandular localization was found in EE throughout the menstrual cycle (Figure 1A). OE and DIE samples showed moderate to strong periostin expression levels in the stroma (Figure 1A). To analyze the levels of significance, the histo 

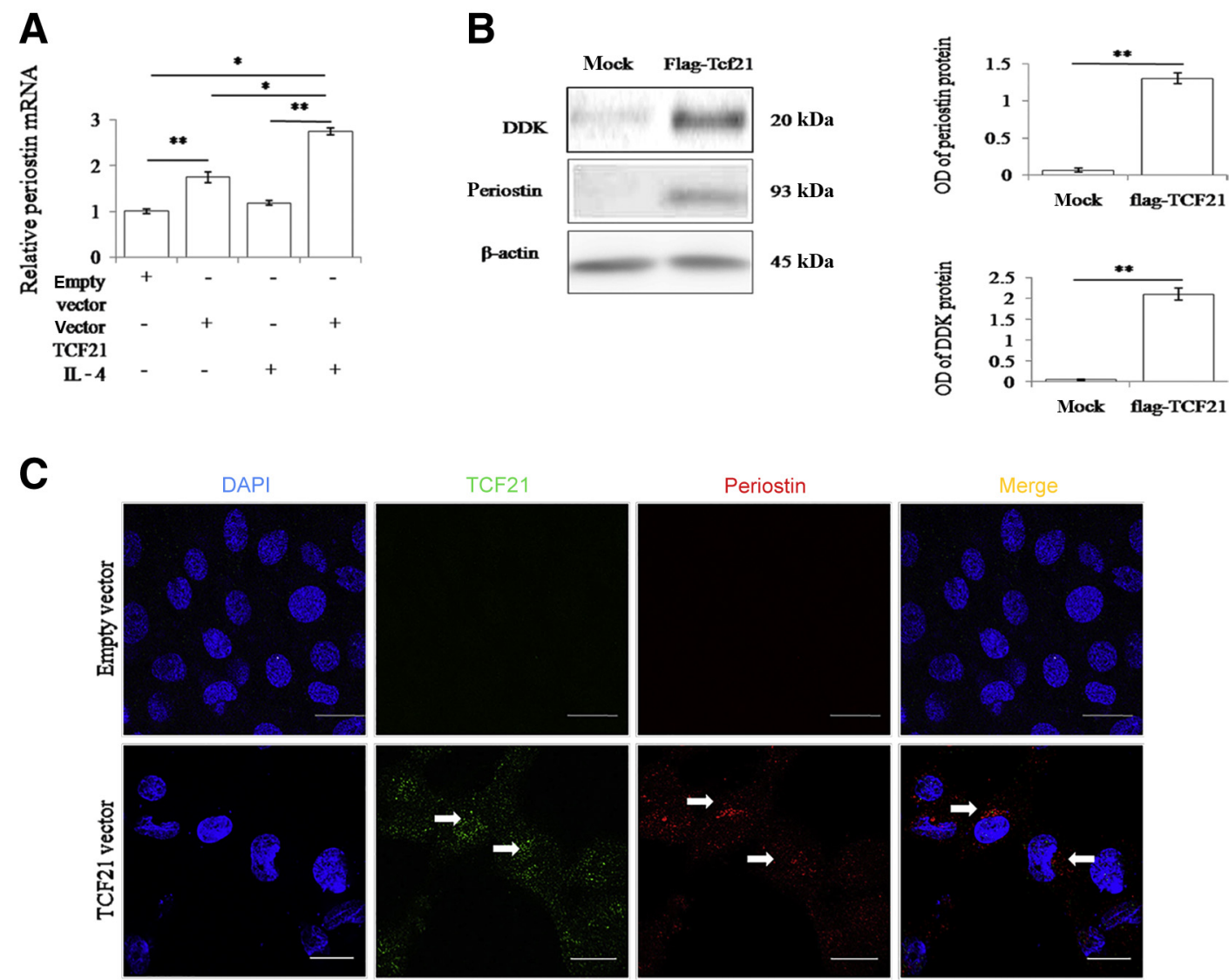

Figure 8 Effect of TCF21 overexpression on periostin. A: mRNA levels of periostin in endometrial stromal cell (ESC) after transfection with TCF21/DDKtagged plasmid (48 hours). B: Western blot analysis of the levels of periostin protein in ESC after TCF21/DDK-tagged plasmid transfection (48 hours). C: Immunocytochemical analysis of the colocalization of TCF21 and periostin after TCF21/DDK-tagged plasmid transfection in ESC. Arrows indicate TCF21transfected cells with induced production of periostin. Data are expressed as means \pm SD. $n=3(\mathbf{A}-\mathbf{C}) .{ }^{*} P<0.05,{ }^{* *} P<0.01$. Scale bars $=20 \mu \mathrm{m}(\mathbf{C})$. Original magnification, $\times 100$ (C).

score was calculated (Figure 1B). The level of periostin was significantly higher in DIE samples compared with that in OE, $\mathrm{EE}$, and NE samples $(P<0.0001)$; it was negative in NE, whereas there was significance between any two groups $(P<0.001)$. To evaluate the expression of periostin mRNA level in the samples of women with or without endometriosis, qPCR was performed with the mRNA samples obtained from the cells cultured for several passages. The highest level of periostin mRNA was observed in DSC, with significance between any two groups (Figure 1C). WB analysis revealed that periostin protein level was in line with the mRNA expression level (Figure 1D). These findings suggest that periostin is exclusively featured in women with endometriosis. Typically, more fibrosis was observed in OE compared with EE, and further fibrosis was encountered in DIE compared with $\mathrm{OE}{ }^{40}$

\section{TCF21 Expression}

Previous studies have reported the interaction between TCF21 and periostin. ${ }^{34,35}$ To assess this interaction in endometriosis, TCF2 1 protein distribution was analyzed by immunohistochemistry in NE, EE, OE, and DIE samples.
In NE, TCF21 was expressed neither in proliferative nor in secretory phases of the menstrual cycle (Figure 2A). Weak expression with mainly glandular localization was found in EE in both phases of the menstrual cycle (Figure 2A). In $\mathrm{OE}$, moderate expression of TCF21 protein was found in the stroma (Figure 2A). Similarly, DIE showed mainly stromal pattern of TCF21 protein distribution; however, the expression level was stronger (Figure 2A). The histo score showed significant differences $(P<0.001)$ in the levels of TCF21 within any two groups, with the highest level in DIE compared with OE, EE, and NE $(P<0.0001)$ (Figure 2B). qPCR with the mRNA samples obtained from the cell cultures was performed to assess the relative expression of TCF21 mRNA in the samples of women with or without endometriosis. Similar to periostin, the highest level of TCF21 mRNA was observed in DSC compared with CSC, eutopic ESC, and ESC, with significance between any two groups (Figure 2C). These findings are in line with the results of $\mathrm{WB}$ analysis of the levels of TCF21 protein (Figure 2D). These data suggest that TCF21 is uniquely expressed in women with endometriosis. 


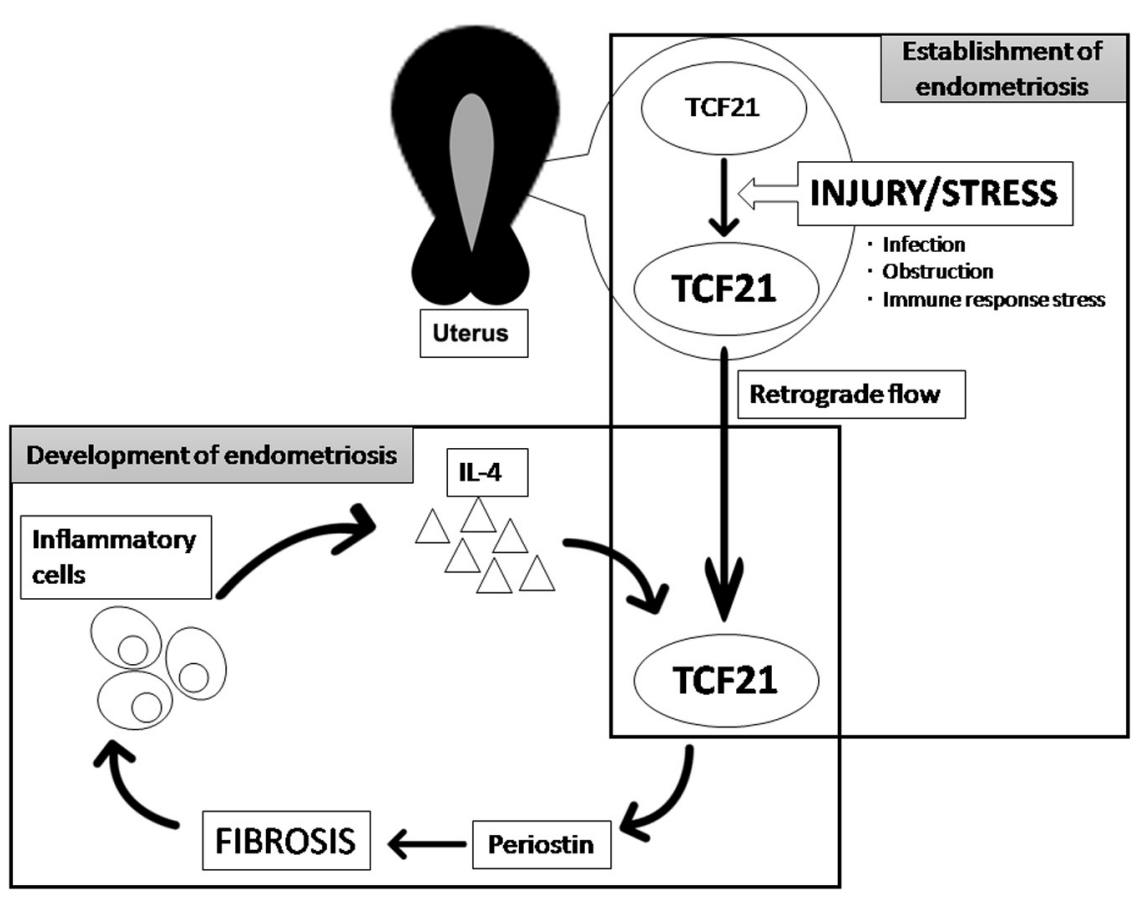

Figure 9 Schematic view of possible interaction between cytokines, TCF21, and periostin in a vicious cycle of coactivation in endometriosis. TCF21 is ubiquitously expressed in all cells; and only after injury or stress, it becomes aberrantly activated. The activated TCF21 then regulates the expression of genes promoting proliferation, survival, and epithelial-mesenchymal transition. With retrograde flow, the TCF21-activated endometrium may attach to the ectopic tissue, leading to a stromal invasion of TCF21-activated endometrium and its induction of periostin production. Periostin contributes to fibrosis and activates immune cells to produce proinflammatory cytokines (eg, IL-4) to further expand inflammation. The resulting fibrosis and inflammation construct the vicious cycle of the pathogenesis of endometriosis.

The Effect of Cytokines on the Expression of Periostin and TCF21

The fibrotic aspect of endometriosis was studied so far; inflammation also greatly contributes to fibrosis. Therefore, the influence of the type 2 helper T-cell profibrotic cytokines (IL-4, IL-13, and TGF- $\beta 1$ ) was examined on periostin and TCF21 expression. These cytokines increased periostin and TCF21 mRNA levels (Figure 3). Specifically, periostin and TCF21 were most significantly induced in DSC, with significance between any two cell types. Furthermore, to investigate the interaction between cytokines and periostin, cytokines and TCF21, and cytokines, periostin, and TCF21 expression, immunofluorescence staining was performed. As hypothesized, there was no signal of periostin and cytokines (Figure 4), TCF21 and cytokines (Figure 5), and periostin, TCF21, and cytokines (Figure 6) in NE samples, in either secretory or proliferative phases of the menstrual cycle. In EE, cytokines, periostin, and TCF21 were weakly expressed in both phases; and they were colocalized in the areas of their expression, which was evaluated on the basis of the color change on the merged images (Figures 4, 5, and 6). Moderate to strong stromal signal and colocalization were detected in OE and DIE samples, respectively (Figures 4, 5, and 6). The signals of periostin, TCF21, and cytokines in OE and DIE were more intensive and more abundant than in EE and NE samples. Therefore, they were evaluated as being moderate and strong signals. These results suggest that cytokines, periostin, and TCF21 expression increased with their colocalization.

\section{Transient Knockdown and Overexpression of TCF21}

Colocalization of TCF21 and periostin (Figure 6) suggested that TCF 21 may be an upstream regulatory gene of periostin in the pathogenesis of endometriosis. Thus, siRNAmediated knockdown of TCF21 was performed to check its possible influence on periostin retention. As hypothesized, qPCR showed that TCF21 siRNA transiently knocked down not only TCF21 (Figure 7A) but also periostin mRNA expression in CSC and DSC (Figure 7B). WB analysis revealed similar changes in the periostin expression levels of CSC and DSC (Figure 7C). Moreover, siTCF21 significantly suppressed IL-4-induced elevation of periostin and TCF21 (Figure 7D). Successful knockdown of periostin by TCF21 siRNA suggested that TCF21 transfection in ESC, which was negative for both TCF21 and periostin, may evoke periostin expression. qPCR and WB analysis showed that ESC transfected with TCF21 plasmid expressed both TCF21 and periostin (Figure 8, A and B). Transfected TCF21 was found to be localized in the nucleus, whereas periostin was found in the cytoplasm of ESC (Figure 8C). These findings inferred that $T C F 21$ can effectively regulate periostin during the development of endometriosis.

\section{Discussion}

Endometriosis is one of the major causes of infertility among women in reproductive age. Despite continuous biomedical research efforts, the pathogenesis of endometriosis remains unclear. Fibrogenesis and fibroproliferation are important stages for the formation of endometriotic 
lesions. Some studies have already emphasized the consistent presence of fibrosis and myofibroblasts in endometriotic lesions and their importance in the pathogenesis of the disease. $^{41-43}$ Myofibroblasts are usually activated in response to injury. Their intent is to repair damaged ECM. Myofibroblasts can be dispatched from various cellular lineages, including endothelial cells undergoing endothelialto-mesenchymal transition, tissue resident fibroblasts, vascular smooth muscle cells, and epithelial cells after EMT. ${ }^{44}$ Periostin acts as a switch in the assembly of all ECM proteins, participating in cell adhesion, differentiation, and organization of ECM in case of tissue damage. ${ }^{45}$ Particularly, its direct binding to fibronectin in the endoplasmic reticulum of fibroblastic cells supports the suggested role of periostin in the protein secretory pathway. ${ }^{19}$ There is a detailed description of the role of periostin in ophthalmology, dermatology, respiratory processes, otolaryngology, and oncology, where it is activated in response to local inflammation triggered by cytokines, supported by integrins and downstream signals. ${ }^{45}$ However, the possible downstream and upstream regulatory genes of periostin in endometriosis are not fully described yet. Hence, periostin is gaining a great interest in relation to fibrotic processes in endometriosis.

In the present study, the interaction between TCF 21 and periostin was clearly demonstrated in the samples of women with or without endometriosis. TCF21 and periostin levels were elevated in the samples from patients with $\mathrm{OE}$ and DIE, supporting their role in the pathogenesis of endometriosis. In previous reports demonstrating the distribution of periostin, the endometriotic samples were not considered separately as OE/CSC and DIE/DSC types. ${ }^{5,28,36}$ For the first time, the localization of both, TCF21 and periostin proteins, was analyzed by separating the samples into NE, $\mathrm{EE}, \mathrm{OE}$, and DIE; and a correlation was found between severe fibrosis and strong expression of both proteins. ${ }^{40}$ Both TCF21 and periostin could be involved in the fibrotic proliferation of endometriosis. The interaction between TCF 21 and periostin was then studied. Treatment with siTCF21 significantly decreased the expression of periostin. After transfection of ESC, formally negative for TCF21 and periostin, with DDK-tagged TCF21 vector plasmid, ESC expressed both proteins. These findings suggested that periostin may be regulated by TCF 21 in endometriosis, once TCF 21 is activated.

The pathogenesis of endometriosis consists of the vicious cycle of inflammation and fibrosis. Therefore, type 2 helper T-cell cytokines that promote fibrosis were studied. An increased level of periostin is induced by type 2 helper T-cell anti-inflammatory cytokines, mainly IL-4, IL-13, and TGF- $\beta$ in asthma, myocardial infarction, and some types of tumors. ${ }^{46}$ As expected, in this research, the same cytokines increased TCF21 levels, leading to elevated periostin levels. These profibrotic cytokines were found to be closely localized with both TCF21 and periostin in endometriotic tissues, and they were not expressed in the tissue samples of women without endometriosis. These results suggest that TCF 21 may be activated in normal endometrium before the pathologic process leading to endometriosis starts. Howev$\mathrm{er}$, it is not clear whether the activation of cytokines precedes the trigger of TCF 21 or whether TCF 21 attracts the cytokines once it is switched on, followed by a vicious cycle of coactivation (Figure 9). Although, in other cell types, TCF21 and periostin have nuclear and cytoplasmic localization, respectively, ${ }^{17,33}$ there are no previous studies showing the cellular localization of these proteins in endometriosis. Periostin is generated inside the cell, even if it is classified as one of the ECM proteins. ${ }^{47}$ In our findings, periostin was localized in the cytoplasm of stromal cells, close to TCF21, which is situated in the nuclear compartment of the cells.

TCF21 is ubiquitously expressed in all cells and remains inactive under normal conditions. Only after injury or stress, it becomes aberrantly activated. ${ }^{34-36}$ Xiang et al ${ }^{34}$ demonstrated that stress caused by myocardial infarction induced the activation of TCF21 in cardiac fibroblasts. The activated TCF21 then regulates the expression of genes promoting proliferation, survival, and EMT. ${ }^{34-36}$ The same injury or stress could happen in the uterus. Bacterial endotoxin (lipopolysaccharide) has been reported to regulate the proinflammatory response in the pelvis and growth of endometriosis via the lipopolysaccharide/toll-like receptor 4 cascade ${ }^{48}$ Cicinelli et $\mathrm{al}^{49}$ reported that intrauterine microbes causing chronic endometritis may be the initial factor for developing endometriosis. The increased prevalence of endometriosis reported in women with Herlyn-WernerWunderlich syndrome with menstrual blood outflow obstruction has been suggested to be caused by the inability of the immune response to remove the debris. ${ }^{50}$ Such infectious injury and immune response stress may contribute to the activation of TCF21 in the normal uterus, leading to endometriotic predisposition. With retrograde flow, the TCF21-activated endometrium could attach to the ectopic tissue, leading to a stromal invasion of TCF21-activated endometrium and its induction of periostin production. According to some disputes regarding endometriosis development, DIE is not formed via retrograde menstruation, like other endometriotic lesions. Our findings showed that although both ectopic lesions-DIE and OE-showed high expressions of TCF21 and periostin, DIE showed higher expressions than OE. Therefore, all types of lesions might have retrograde menstruation as an initial triggering factor that should necessarily be accompanied by favorable endocrine and metabolic environment, EMT, altered immunity, and inflammatory responses. ${ }^{51}$

Along with the need to better understand the pathophysiology of endometriosis, the need for a reliable diagnostic biomarker for endometriosis is imperative. It is highly likely that, instead of a single biomarker, a group of biomarkers, including TCF21 and periostin, will provide improved diagnostic performance and minimize falsepositive results during differential diagnosis. 
Protein and mRNA analyses were performed on stromal cells that were cultured from the tissue samples. Therefore, there might be a change in gene expression during in vitro culture. However, such changes were not examined in this study.

\section{Conclusions}

This study highlights the importance of $T C F 21$ in periostin regulation in vitro. TCF 21 may be a key regulator for switching off periostin, which retains the scaffold of all ECM proteins necessary to cause fibrosis in endometriotic lesions. In vitro data suggest that TCF21 may become a novel preventive and therapeutic target as well as a reliable biomarker in endometriosis.

\section{References}

1. Zhang Q, Duan J, Olson M, Fazleabas A, Guo SW: Cellular changes consistent with epithelial-mesenchymal transition and fibroblast-tomyofibroblast transdifferentiation in the progression of experimental endometriosis in baboons. Reprod Sci 2016, 23:1409-1421

2. Giudice LC, Kao LC: Endometriosis. Lancet 2004, 364:1789-1799

3. Bulun SE: Endometriosis. N Engl J Med 2009, 360:268-279

4. Sampson JA: Metastatic or embolic endometriosis, due to the menstrual dissemination of endometrial tissue into the venous circulation. Am J Pathol 1927, 3:93-110.43

5. Liu H, Lang JH: Is abnormal eutopic endometrium the cause of endometriosis? the role of eutopic endometrium in pathogenesis of endometriosis. Med Sci Monit 2011, 17:RA92-RA99

6. Zhang Q, Dong P, Liu X, Sakuragi N, Guo SW: Enhancer of Zeste homolog 2 (EZH2) induces epithelial-mesenchymal transition in endometriosis. Sci Rep 2017, 7:6804

7. Yang YM, Yang WX: Epithelial-to-mesenchymal transition in the development of endometriosis. Oncotarget 2017, 8:41679-41689

8. Iwase A, Kotani T, Goto M, Kobayashi H, Takikawa S, Nakahara T, Nakamura T, Kondo M, Bayasula, Nagatomo Y, Kikkawa F: Possible involvement of CD10 in the development of endometriosis due to its inhibitory effects on CD44-dependent cell adhesion. Reprod Sci 2014, 21:82-88

9. Yang H, Liu J, Fan Y, Guo Q, Ge L, Yu N, Zheng X, Dou Y, Zheng S: Associations between various possible promoter polymorphisms of MMPs genes and endometriosis risk: a meta-analysis. Eur J Obstet Gynecol Reprod Biol 2016, 205:174-188

10. Cao Y, Ye Q, Zhuang M, Xie S, Zhong R, Cui J, Zhou J, Zhu Y, Zhang T, Cao L: Ginsenoside Rg3 inhibits angiogenesis in a rat model of endometriosis through the VEGFR-2-mediated PI3K/Akt/mTOR signaling pathway. PLoS One 2017, 12:e0186520

11. Chen ZZ, Gong X: Effect of Hua Yu Xiao Zheng decoction on the expression levels of vascular endothelial growth factor and angiopoietin-2 in rats with endometriosis. Exp Ther Med 2017, 14: $5743-5750$

12. Qu J, Zhu Y, Wu X, Zheng J, Hou Z, Cui Y, Mao Y, Liu J: Smad3/4 binding to promoter II of P450arom so as to regulate aromatase expression in endometriosis. Reprod Sci 2017, 24:1187-1194

13. Shi LB, Zhou F, Zhu HY, Huang D, Jin XY, Li C, Dai Y, Pan YB, Zhang SY: Transforming growth factor beta1 from endometriomas promotes fibrosis in surrounding ovarian tissues via $\mathrm{Smad} 2 / 3$ signaling. Biol Reprod 2017, 97:873-882

14. Li MQ, Li HP, Meng YH, Wang XQ, Zhu XY, Mei J, Li DJ: Chemokine CCL2 enhances survival and invasiveness of endometrial stromal cells in an autocrine manner by activating Akt and MAPK/Erk1/2 signal pathway. Fertil Steril 2012, 97:919-929
15. Burney RO, Giudice LC: Pathogenesis and pathophysiology of endometriosis. Fertil Steril 2012, 98:511-519

16. Falcone T, Flyckt R: Clinical management of endometriosis. Obstet Gynecol 2018, 131:557-571

17. Sugiura T, Takamatsu H, Kudo A, Amann E: Expression and characterization of murine osteoblast-specific factor 2 (OSF-2) in a baculovirus expression system. Protein Expr Purif 1995, 6: 305-311

18. Elliott CG, Hamilton DW: Deconstructing fibrosis research: do profibrotic signals point the way for chronic dermal wound regeneration? J Cell Commun Signal 2011, 5:301-315

19. Kii I, Nishiyama T, Li M, Matsumoto K, Saito M, Amizuka N, Kudo A: Incorporation of tenascin-C into the extracellular matrix by periostin underlies an extracellular meshwork architecture. J Biol Chem 2010, 285:2028-2039

20. Snider P, Hinton RB, Moreno-Rodriguez RA, Wang J, Rogers R, Lindsley A, Li F, Ingram DA, Menick D, Field L, Firulli AB, Molkentin JD, Markwald R, Conway SJ: Periostin is required for maturation and extracellular matrix stabilization of noncardiomyocyte lineages of the heart. Circ Res 2008, 102:752-760

21. Suzuki H, Amizuka N, Kii I, Kawano Y, Nozawa-Inoue K, Suzuki A, Yoshie H, Kudo A, Maeda T: Immunohistochemical localization of periostin in tooth and its surrounding tissues in mouse mandibles during development. Anat Rec A Discov Mol Cell Evol Biol 2004 281:1264-1275

22. Norris RA, Moreno-Rodriguez R, Hoffman S, Markwald RR: The many facets of the matricelluar protein periostin during cardiac development, remodeling, and pathophysiology. J Cell Commun Signal 2009, 3:275-286

23. Rios H, Koushik SV, Wang H, Wang J, Zhou HM, Lindsley A, Rogers R, Chen Z, Maeda M, Kruzynska-Frejtag A, Feng JQ, Conway SJ: Periostin null mice exhibit dwarfism, incisor enamel defects, and an early-onset periodontal disease-like phenotype. Mol Cell Biol 2005, 25:11131-11144

24. Bozyk PD, Bentley JK, Popova AP, Anyanwu AC, Linn MD, Goldsmith AM, Pryhuber GS, Moore BB, Hershenson MB: Neonatal periostin knockout mice are protected from hyperoxia-induced alveolar simplication. PLoS One 2012, 7:e31336

25. Yuyama N, Davies DE, Akaiwa M, Matsui K, Hamasaki Y, Suminami Y, Yoshida NL, Maeda M, Pandit A, Lordan JL, Kamogawa Y, Arima K, Nagumo F, Sugimachi M, Berger A, Richards I, Roberds SL, Yamashita T, Kishi F, Kato H, Arai K, Ohshima K, Tadano J, Hamasaki N, Miyatake S, Sugita Y, Holgate ST, Izuhara K: Analysis of novel disease-related genes in bronchial asthma. Cytokine 2002, 19:287-296

26. Oka T, Xu J, Kaiser RA, Melendez J, Hambleton M, Sargent MA, Lorts A, Brunskill EW, Dorn GW, Conway SJ, Aronow BJ, Robbins J, Molkentin JD: Genetic manipulation of periostin expression reveals a role in cardiac hypertrophy and ventricular remodeling. Circ Res 2007, 101:313-321

27. Wong VW, Rustad KC, Akaishi S, Sorkin M, Glotzbach JP, Januszyk M, Nelson ER, Levi K, Paterno J, Vial IN, Kuang AA, Longaker MT, Gurtner GC: Focal adhesion kinase links mechanical force to skin fibrosis via inflammatory signaling. Nat Med 2011, 18: $148-152$

28. Shen L, Liu P, Zhang P, Zhang X, Cui J: Characterization of periostin expression in human endometrium and endometriotic lesions. Gynecol Endocrinol 2012, 28:815-818

29. Xu X, Zheng Q, Zhang Z, Zhang X, Liu R, Liu P: Periostin enhances migration, invasion, and adhesion of human endometrial stromal cells through integrin-linked kinase 1/Akt signaling pathway. Reprod Sci 2015, 22:1098-1106

30. Zheng Q, Lu J, Li R, Hu C, Liu P: Elevated periostin in serum and peritoneal washing fluids as potential biomarkers for endometriosis. Gynecol Endocrinol 2016, 32:900-903

31. Zheng QM, Lu JJ, Zhao J, Wei X, Wang L, Liu PS: Periostin facilitates the epithelial-mesenchymal transition of endometrial epithelial 
cells through ILK-Akt signaling pathway. Biomed Res Int 2016, 2016:9842619

32. Logan PC, Yango P, Tran ND: Endometrial stromal and epithelial cells exhibit unique aberrant molecular defects in patients with endometriosis. Reprod Sci 2017, 25:140-159

33. Robb L, Mifsud L, Hartley L, Biben C, Copeland NG, Gilbert DJ, Jenkins NA, Harvey RP: Epicardin: a novel basic helix-loop-helix transcription factor gene expressed in epicardium, branchial arch myoblasts, and mesenchyme of developing lung, gut, kidney, and gonads. Dev Dyn 1998, 213:105-113

34. Xiang FL, Fang M, Yutzey KE: Loss of $\beta$-catenin in resident cardiac fibroblasts attenuates fibrosis induced by pressure overload in mice. Nat Commun 2017, 8:712

35. Kanisicak O, Khalil H, Ivey MJ, Karch J, Maliken BD, Correll RN, Brody MJ, Lin SCJ, Aronow BJ, Tallquist MD, Molkentin JD: Genetic lineage tracing defines myofibroblast origin and function in the injured heart. Nat Commun 2016, 7:12260

36. Wu PL, Zhou Y, Zeng C, Li X, Dong ZT, Zhou YF, Bulun SE, Xue Q: Transcription factor 21 regulates expression of ER $\beta$ and SF-1 via upstream stimulatory factor-2 in endometriotic tissues. Biochim Biophys Acta Gene Regul Mech 2018, 1891:706-717

37. Gaetje R, Holtrich U, Karn T, Cikrit E, Engels K, Rody A, Kaufmann M: Characterization of WNT7A expression in human endometrium and endometriotic lesions. Fertil Steril 2007, 88:1534-1540

38. Zhang H, Xue J, Li M, Zhao X, Wei D, Li C: Metformin regulates stromal-epithelial cells communication via Wnt2/ $\beta$-catenin signaling in endometriosis. Mol Cell Endocrinol 2015, 413:61-65

39. Matsuzaki S, Darcha C: In vitro effects of a small-molecule antagonist of the Tcf/ß-catenin complex on endometrial and endometriotic cells of patients with endometriosis. PLoS One 2013, 8:e61690

40. Liu X, Zhang Q, Guo SW: Histological and immunohistochemical characterization of the similarity and difference between ovarian endometriomas and deep infiltrating endometriosis. Reprod Sci 2018, 25:329-340
41. Anaf V, Simon P, Fayt I, Noel J: Smooth muscles are frequent components of endometriotic lesions. Hum Reprod 2000, 15: $767-771$

42. Barcena de Arellano ML, Gericke J, Reichelt U, Okuducu AF, Ebert AD, Chiantera V, Schneider A, Mechsner S: Immunohistochemical characterization of endometriosis-associated smooth muscle cells in human peritoneal endometriotic lesions. Hum Reprod 2011, 26:2721-2730

43. Zhang Q, Duan J, Liu X, Guo SW: Platelets drive smooth muscle metaplasia and fibrogenesis in endometriosis through epithelialmesenchymal transition and fibroblast-to-myofibroblast transdifferentiation. Mol Cell Endocrinol 2016, 428:1-16

44. Vigano P, Candiani M, Monno A, Giacomini E, Vercellini P, Somigliana P: Time to redefine endometriosis including its profibrotic nature. Hum Reprod 2018, 33:347-352

45. Kudo A, Kii I: Periostin function in communication with extracellular matrices. J Cell Commun Signal 2017, 12:301-308

46. Izuhara $\mathrm{K}$, Nunomura $\mathrm{S}$, Nanri $\mathrm{Y}$, Ogawa $\mathrm{M}$, Ono J, Mitamura $\mathrm{Y}$, Yoshihara T: Periostin in inflammation and allergy. Cell Mol Life Sci 2017, 74:4293-4303

47. Kudo A: Introductory review: periostin-gene and protein structure. Cell Mol Life Sci 2017, 74:4259-4268

48. Khan KN, Fujishita A, Hiraki K, Kitajima M, Nakashima M, Fushiki S, Kitawaki J: Bacterial contamination hypothesis: a new concept in endometriosis. Reprod Med Biol 2018, 17:125-133

49. Cicinelli E, Trojano G, Mastromauro M, Vimercati A, Marinaccio M, Mitola PC, Resta L, de Ziegler D: Higher prevalence of chronic endometritis in women with endometriosis: a possible ethiopathogenetic link. Fertil Steril 2017, 108:289-295

50. Tong J, Zhu L, Chen N, Lang J: Endometriosis in association with Herlyn-Werner-Wunderlich syndrome. Fertil Steril 2014, 102: 790-794

51. Zondervan KT, Becker CM, Koga K, Missmer SA, Taylor RN, Vigano P: Endometriosis. Nat Rev Dis Primers 2018, 4:9 